\title{
GPs' perceived barriers to their involvement in caring for patients with HIV: a questionnaire-based study
}

\author{
Helen Defty, Helen Smith, Michelle Kennedy, Nicky Perry and Martin Fisher
}

\begin{abstract}
Background

Since the introduction of highly active antiretroviral therapy (HAART) in the late 1990s, individuals with HIV are living longer and beginning to experience the chronic diseases of ageing. Alongside side effects of HAART therapy, these may be better managed in the community by generalists rather than by HIV specialists.
\end{abstract}

Aim

To explore GPs' current perceptions of barriers to their involvement in managing patients with HIV.

Design of study

Postal questionnaire.

Setting

Practices within a primary care trust in the south of

England.

Method

The questionnaire included 24 statements relating to potential structural and attitudinal barriers to GPs' involvement in the care of patients with HIV.

\section{Results}

Seventy-one per cent (124/174) of GPs responded; 93\% $(n=115)$ of responders were comfortable managing other illness in HIV patients, and 60\% $(n=$ 74) agreed that GPs rather than specialists should manage common chronic problems such as hypertension or diabetes. Specialist prescribing was commonly identified as a barrier, specifically: difficulty keeping up to date, knowledge of HAART side effects, and drug interactions. The majority of responders $(67 \%$ [83]) viewed patients as preferring their HIV illness to be managed by specialists.

\section{Conclusion}

There is now the opportunity and potential to relocate the management of chronic disease in patients with HIV to GPs. Factors such as lack of time, experience, and training were identified as barriers to caring for patient with HIV in the pre-HAART era; these factors continue to challenge some GPs. Additional barriers are the complexity of HAART regimes and inadequate reimbursement. Addressing these barriers is necessary if services are to be reconfigured for people with HIV.

Keywords

antiretroviral therapy; family practice; highly active; HIV.

\section{INTRODUCTION}

Since the introduction in the late 1990s of highly active antiretroviral therapy (HAART), individuals with HIV are living longer and may enjoy near-normal life expectancy. Thus, people living with HIV will, in time, start to experience the chronic diseases of ageing. These chronic conditions, alongside the side effects of HAART therapy, may be better managed in the community by generalists rather than by HIV specialists. ${ }^{1,2}$ The British HIV Association (BHIVA) recommends increased GP involvement in caring for patients with HIV. ${ }^{3}$ However, studies from the mid1990s, prior to the availability of HAART, found that GPs perceived significant barriers to caring for patients with HIV, including a lack of time, knowledge, experience, and training. ${ }^{4,5}$ The aim of the questionnaire administered in this study was to explore GPs' current perceptions of the barriers to their involvement in managing patients with HIV.

\section{METHOD}

The questionnaire was developed following review of two pre-HAART studies, ${ }^{4,5}$ and in consultation with

H Defty, MA, BMBS, FY2 trainee, South Thames Foundation School, London. M Kennedy, BA(Mod), BMBS, FY2 trainee, North West Thames Foundation School, Imperial College London, London. H Smith, MSc, DM, MRCGP, FFPHM, chair of primary care, Brighton and Sussex Medical School. $N$ Perry, RN MSc, research manager; M Fisher, BSc, FRCP, consultant, Department of HIV of Genitourinary Medicine, Brighton and Sussex University Hospitals Trust.

Address for correspondence

Helen Smith, Head of Division of Public Health and Primary Care, Room 319, Mayfield House, University of Brighton, Falmer BN1 9PH. E-mail: h.e.smith@bsms.ac.uk

Submitted: 30 August 2009; Editor's response: 4 November 2009; final acceptance: 7 January 2010.

(c)British Journal of General Practice 2010; 60: 348-351.

DOI: 10.3399/bjgp10X501840 
experts in primary care, HIV medicine, and questionnaire design. The questionnaire was piloted with six GPs purposively sampled from practices with low, medium, and high numbers of patients with HIV. Piloting directed improvements in layout, question phrasing, and content. The refined questionnaire included 24 statements relating to potential structural and attitudinal barriers to GPs' involvement in the care of HIV patients. The response to each item was measured using a five-point Likert scale; the response options were 'strongly agree', 'agree', 'undecided', 'disagree', and 'strongly disagree'. GPs were able to make additional relevant comments in free text at the end of the questionnaire. Demographic and practice variables were also collected.

The prevalence rate of HIV within Brighton and Hove Primary Care trust (PCT) is seven per 1000; excluding London PCTs, this is the highest HIV rate within an English PCT. ${ }^{6}$ All GPs on the Brighton and Hove PCT register were sent a postal questionnaire accompanied by a covering letter, response card and stamped-addressed envelope (March 2007). Separating personal details from the questionnaire by using a response card allowed follow-up of nonresponders while maintaining confidentiality (Dillman's method). ${ }^{7}$ As an incentive, responders were placed in a prize-draw for restaurant vouchers. ${ }^{8}$ Non-responders were followed-up by post at 2 weeks; data collection stopped at 8 weeks.

\section{Analysis}

Data were analysed using SPSS (version 12). Likert responses were treated as ordinal variables and analysed using non-parametric tests. Kendall's $\tau$ test, accounting for tied rank, was used to assess correlations between question responses. ${ }^{9}$ The Mann-Whitney $U$ test was used to investigate association of experience (defined as treating $>10$ patients with HIV) and training with other responses. For analysis, responses were combined, that is, 'agree' and 'strongly agree' were collated to represent agreement, and a similar combination was used for responses of disagreement. Free-text comments underwent thematic analysis.

\section{How this fits in}

Since the introduction of highly active antiretroviral therapy (HAART), individuals with HIV are living longer; the chronic diseases of ageing, alongside the side effects of HAART therapy, may be better managed in the community by GPs rather than by HIV specialists. In the pre-HAART era, there were significant barriers to GPs caring for patients with HIV; now almost all GPs feel comfortable managing other illness in patients with HIV. One-third of GPs are interested in increasing their involvement in the care of patients with HIV. The complexity of HAART regimes, their interactions and side effects, and inadequate reimbursement are identified as barriers to providing enhanced care.

\section{RESULTS}

\section{Response rate and sample characteristics}

The overall questionnaire response rate was $71 \%$ (124/174). Sixty responders claimed to work at a locally enhanced service practice; 73 GPs work at such practices (response rate $82 \%$ ). Only two GPs (2\%) had never cared for someone with HIV. Nine per cent $(n=11)$ of GPs had previously held a genitourinary medicine or HIV post. Sixty-one per cent $(n=76)$ had attended an HIV training event and $48 \%(n=60)$ of responders worked at practices providing the HIV locally enhanced service.

\section{Barriers to care}

Examples of statements relating to structural and attitudinal barriers, with their corresponding responses, are shown in Tables 1 and 2. Ninety-three per cent $(n=115)$ of responders were comfortable managing other illness in patients with HIV. However, $23 \%(n=28)$ did not feel confident distinguishing between HIV illness and other illness. Sixty per cent ( $n=74)$ of responders agreed that GPs rather than specialists should manage problems such as hypertension, hyperlipidaemia, or diabetes. Specialist prescribing was commonly identified as a barrier, specifically: difficulty keeping up to date with anti-HIV therapies $(79 \%[n=98])$, knowledge of HAART side effects $(72 \%[n=89])$, and drug interactions $(46 \%[n=57])$. Other important structural barriers included inadequate

Table 1. Examples of statements on structural barriers to care in general practice of patients with HIV.

\begin{tabular}{|c|c|c|c|}
\hline Statements & Agree, \% (n) & Undecided,\% ( $n)$ & Disagree, \% (n) \\
\hline 'I am sure I can distinguish between HIV illness and other illnesses' & $30(37)$ & $47(58)$ & $23(28)$ \\
\hline 'I feel there is currently adequate training offered to allow GPs to look after HIV patients' & $28(34)$ & $30(37)$ & $43(53)$ \\
\hline 'I do not feel we should take on any extra care of HIV patients unless we get specific reimbursement & t' $54(67)$ & $29(36)$ & $14(18)$ \\
\hline 'I feel there is adequate communication between HIV specialists and primary care' & $47(58)$ & $20(24)$ & $33(42)$ \\
\hline
\end{tabular}

Maximum number of missing responses for any statement was $2 \%(n=3)$. 
Table 2. Examples of statements on attitudinal barriers to care in general practice of patients with HIV.

Statements

Agree, \% (n) Undecided,\% (n) Disagree, \% (n)

\begin{tabular}{|c|c|c|c|}
\hline 'I feel uncomfortable looking after a patient whose sexuality is different from my own' & $4(5)$ & $1(1)$ & $94(117)$ \\
\hline 'My patients prefer to have their HIV illness managed by specialists' & $67(83)$ & $30(37)$ & $2(3)$ \\
\hline 'My patients with HIV prefer to have all their health issues managed by specialists' & $20(25)$ & 40 (49) & $40(50)$ \\
\hline $\begin{array}{l}\text { 'Historically HIV patients have been looked after mainly in secondary care, but their basic } \\
\text { care should now be moved over to primary care' }\end{array}$ & $29(36)$ & $45(56)$ & $25(31)$ \\
\hline
\end{tabular}

care should now be moved over to primary care'

Maximum number of missing responses for any statement was $2 \%(n=3)$.

communication between primary and secondary care, lack of specific reimbursement, insufficient experience, inadequate training, and shortage of time. The majority of responders viewed patients as preferring their HIV illness to be managed by specialists $(67 \%$ [ $n=83])$. Twenty per cent $(n=25)$ felt their patients with HIV prefer to have all health issues managed by specialists.

\section{Future involvement}

Thirty-four per cent $(n=42)$ of responders expressed definite interest in taking on more HIV care in the future, excluding initiating antiretroviral therapy; but only one GP expressed an interest in initiating antiretrovirals. There was a strong significant negative relationship between identifying time as a barrier and interest in taking on more HIV care in the future $(r=-1.92 ; P=0.012)$. There were no significant relationships between other potentially predictive characteristics of active engagement, including training and HIV experience and interest in increased involvement.

\section{Experience, training, and barriers to care}

GPs who had attended an HIV training course (defined as trained GPs) felt significantly more comfortable in managing HIV-related illness (mean rank 74 versus $50, Z=-4.00 ; P<0.01$ ). Importantly, trained GPs felt more up to date with anti-HIV medications (mean rank 70 versus 48; $Z=-3.66$; $P<0.001$ ) and side effects (mean rank 69 versus 50; $Z=-3.36 ; \quad P<0.001)$. Trained GPs were not significantly different in wanting to increase their involvement in HIV care (mean rank 61 versus 62; $Z$ $=-0.022 ; P=0.98$ ). Similar results were found comparing responses from responders who had more than 10 patients (defined as experienced GPs) than with those who had not.

\section{Free-text results}

Thirty-five responders made free-text comments. Three GPs reinforced the view that 'patients with HIV prefer to be cared for by the specialist units'. One responder felt that specialists have encouraged patients away from primary care by being 'prepared to address non-HIV/GU [genitourinary] problems in their patients'. Emphasis was placed on viewing patients as individuals, and respecting 'patients' wishes' regarding primary or secondary care delivery.

Communication difficulties between primary and secondary care were felt by three responders to be 'compounded by the extra confidentiality of medical information' given to patients with HIV. Positive comments were made concerning support from local HIV specialists and pharmacists. While the benefits of having attended a training course were acknowledged, a need for annual refresher courses was expressed: "HIV care is constantly changing'.

Several responders described how in their practice one colleague cared for the majority of HIV patients: 'Our patients get a good level of care without all of the individual GPs in the practice becoming specialised'. Developing posts for 'GPs with a specialist interest in HIV' was suggested. 'Shared care' between the community and hospital setting was seen as the preferred strategy for future management of stable patients. The benefits of having a practice nurse with HIV training were also expressed.

\section{DISCUSSION}

\section{Summary of main findings}

GPs traditionally have not played a major role in the care of people with HIV in the UK, and patients have often consulted in the HIV clinic for unassociated minor illness. Encouragingly, nearly all responders felt comfortable managing other illness in patients with HIV. However, only a third of GPs felt confident distinguishing other illness from HIV-related illness. If HIV patients are to benefit from this willingness of GPs to take on this generalist role, then there will be a need to clarify the boundaries of involvement, to develop good communication with secondary care (and vice versa), and to establish effective referral pathways.

About a third of GPs expressed an interest in taking on more HIV-specific care in the future. There is certainly the potential for primary care to take greater responsibility for those patients whose 
condition is stable, as is evident from the success of a locally enhanced service established in 2003. Practices involved in the locally enhanced service provide monitoring (adherence to treatment, cardiovascular risks, and mental health), advice (drugs, sexual health, lifestyle, vaccinations, and smoking cessation), management of comorbidities, and annual review for patients with HIV. ${ }^{10}$ There is potential to expand GP involvement further with, for example, monitoring of viral load.

GPs who had attended an HIV training course were less likely to perceive barriers to caring for patients with HIV than those with no specific HIV training. Importantly, this comparison included issues relating to HAART. Similar results were found when comparing experienced GPs (defined as having treated more than 10 patients with HIV) with less-experienced GPs. There was, however, no association between experience or training and interest in increased personal involvement in the care of HIV patients. Experienced or trained GPs may already have a high level of involvement and may have a more realistic view of the time demands involved.

\section{Strengths and limitations of the study}

The study response rate of $71 \%(124 / 174)$ is favourable compared to similar surveys. ${ }^{4,5}$ As found elsewhere, GPs with more HIV patients were more likely to respond to the survey, creating potential non-response bias. ${ }^{4}$ It is recognised that Brighton and Hove is an area of high HIV prevalence, and care is needed in generalising to all GPs, as perceived barriers have been shown to vary with prevalence. ${ }^{4}$ One might expect willingness to be greater in other areas where enhanced services do not already exist. This study has focused only on GPs; the views of other members of the primary care team, specialists, and patients should also be considered. Further research, including qualitative work and discrete choice experiments, is needed in regions of low and high HIV prevalence if GP involvement is to be fully understood and promoted nationally.

\section{Comparison with existing literature}

As in this study, a survey from the pre-HAART era by King et al in 1994-1996, ${ }^{4}$ found lack of time, knowledge, training, and experience to be important barriers to care, and the present survey confirms that these barriers persist. In contrast, GPs are no longer challenged with feeling largely unsupported in caring for patients with HIV and the provision of palliative care; today's challenging issues relate to HAART, particularly being aware of new therapeutic options and maintaining knowledge of drug side effects and interactions.

\section{Implications for clinical practice and future research}

This study suggests the majority of GPs are comfortable managing other illness in patients with HIV. There remains some uncertainty, however, over how to identify other illness from HIV illness. A considerable proportion of GPs are interested in increased involvement in the care of patients with HIV. The complexity of HAART regimes, their interactions and side effects, and inadequate reimbursement are newly identified barriers for GPs. In addition, certain barriers existing pre-HAART are continuing, including lack of time, experience, and training. Understanding these barriers is crucial if GP involvement is to be increased. Interventions to involve GPs more fully may be best targeted predominantly at specific interested GPs. Effective interventions may include training updates focusing on distinguishing HIV illness from other illness and managing patients taking HAART. Adequate financial reimbursement is also key to increased primary care involvement.

\section{Funding body}

Brighton and Sussex Medical School.

Ethics committee

The study was approved by Brighton and Mid Sussex Research Ethics Committee (January 2007), 06/Q1907/97.

\section{Competing interests}

The authors have stated that there are none.

\section{Acknowledgements}

We would like to thank all the GPs who participated in this study.

\section{Discuss this article}

Contribute and read comments about this article on the Discussion Forum: http://www.rcgp.org.uk/bjgp-discuss

\section{REFERENCES}

1. Carr A, Cooper D. Adverse effects of antiretroviral therapy. Lancet 2000; 356(9239): 1423-1430.

2. Mansfield S, Singh S. Who should fill the gap in HIV disease? Lancet 1993; 342(8873): 726-728

3. British HIV Association (BHIVA). Standards for HIV clinical care. March 2007. http://www.bhiva.org/HIVClinicalCare.aspx (accessed 29 Mar 2010).

4. King M, Petchey R, Singh S, et al. The role of the general practitioner in the community care of people with HIV infection and AIDS: a comparative study of high and low-prevalence areas in England. $\mathrm{Br}$ Gen Pract 1998; 48(430): 1233-1236.

5. Gerbert B, Maguire B, Blecker T, et al. Primary care physicians and AIDS - attitudinal and structural barriers to care. JAMA 1991; 266(20): 2837-2842.

6. Health Protection Agency. HIV prevalence by local authority 2007 http://www.hpa.org.uk (accessed 15 Jan 2010).

7. Dillman, D A. Mail and telephone surveys: the total design methods. New York, NY: Wiley-Interscience, 1978.

8. Edwards P, Roberts I, Clarke M, et al. Methods to increase response rates to postal questionnaires. Cochrane Database Syst Rev 2007; (2): MR000008. http://www.cochrane.org/reviews/en/mr000008.html (accessed 15 Jan 2010).

9. Bryman A, Cramer D. Quantitative data analysis with SPSS Release 10 for Windows. Hove: Routledge, 2001

10. Brighton and Hove City Teaching Primary Care Trust (PCT). New general medical services contract: specification for the provision of an enhanced service (HIV in primary care). Reference LES 03. Brighton and Hove: Brighton and Hove PCT, 2005. 